\title{
Prevalência de consumo de álcool entre estudantes de Medicina do Centro Universitário de Brasília
}

\author{
Prevalence of alcohol consumption among medical students at the University Center of \\ Brasília
}

\section{Prevalência de consumo de alcohol entre estudiantes de medicina del Centro Universitário de Brasília}

Rafaela de Andrade Silva Miranda ${ }^{1 *}$, Elisa Vasconcelos de Queiroz ${ }^{1}$, Letícia Lara de Lima ${ }^{1}$, Antonio Garcia Reis Junior¹.

\section{RESUMO}

Objetivo: Estimar a prevalência do consumo de álcool entre estudantes de uma faculdade de Medicina no Distrito Federal. Métodos: Trata-se de estudo transversal descritivo, conduzido com 109 pessoas, de ambos os sexos, matriculados no primeiro ou oitavo semestre de medicina do Centro Universitário de Brasília no ano de 2018. Os participantes responderam via online o instrumento "Alcohol Use Disorders Identification Test", para aferir o perfil çonsumo de álcool e estratificar zonas de risco relacionadas ao consumo. O estudo foi aprovado pelo Comitê de Ética em Pesquisa do UniCEUB. Resultados: Responderam ao questionário 44 alunos (40,4\%), sendo $28(63,3 \%)$ destes cursando o $1^{\circ}$ semestre e $16(36,3 \%)$ cursando o $8^{\circ}$ semestre. Constatou-se que $81,9 \%$ das pessoas consumiam bebidas alcoólicas, sendo $75 \%$ cursando o 1 período e $93,8 \%$ o 8 período. Separando por sexo, na turma do $1^{\circ}$ período, $70,6 \%$ das mulheres e $81,9 \%$ dos homens consumiam bebidas alcoólicas, ao passo que para os alunos do $8^{\circ}$ período tais taxas respectivas foram de $100 \%$ e $80 \%$. Conclusão: A pesquisa revelou alta prevalência de consumo de álcool, notadamente maior para as mulheres ao longo do curso de medicina, e com achados semelhantes a outras realidades nacionais e internacionais.

Palavras-chave: Consumo de álcool, Estudantes de medicina, AUDIT.

\begin{abstract}
Objective: To estimate the prevalence of alcohol consumption among students of a medical school in the Federal District. Methods: This is a descriptive cross-sectional study, conducted with 109 people, of both sexes, enrolled in the first or eighth semester of medicine at the Centro Universitário de Brasília in 2018. Participants answered online the instrument "Alcohol Use Disorders Identification Test", to measure the alcohol consumption profile and stratify risk areas related to consumption. The study was approved by the Research Ethics Committee of UniCEUB. Results: 44 students $(40.4 \%)$ answered the questionnaire, of which $28(63.3 \%)$ were in the 1 st semester and 16 $(36.3 \%)$ were in the 8 th semester. It was found that $81.9 \%$ of people consumed alcoholic beverages, with $75 \%$ attending the 1 st period and $93.8 \%$ attending the 8 th period. Separating by sex, in the 1 st period class, $70.6 \%$ of women and $81.9 \%$ of men consumed alcoholic beverages, whereas for students in the 8th period, these respective rates were $100 \%$ and $80 \%$. Conclusion: The research revealed a high prevalence of alcohol consumption, progressively higher for women over the course of medicine, and with findings similar to other national and international realities.
\end{abstract}

Keywords: Alcohol consumption, Medical students, AUDIT.

\section{RESUMEN}

Objetivo: Estimar la prevalencia de consumo de alcohol entre estudiantes de una facultad de medicina del Brasília. Métodos: Fue un estudio descriptivo, transversal, realizado con 109 personas, de ambos sexos, matriculadas en el primer u octavo semestre de medicina en el Centro Universitário de Brasília en 2018. Los participantes respondieron online el instrumento "Alcohol Use Disorders Identification Test", para medir el perfil de consumo de alcohol y estratificar las áreas de riesgo relacionadas con el consumo. El estudio fue aprobado por el Comité de Ética en Investigación de UniCEUB. Resultados: 44 estudiantes (40,4\%) respondieron el cuestionario, de los cuales $28(63,3 \%)$ estaban en el 1er semestre y $16(36,3 \%)$ en el 8 o semestre. Se encontró que el 81,9\% de las personas consumían bebidas alcohólicas, el $75 \%$ asistía al 1er período y el $93,8 \%$ al 8 período. Separando por sexo, en la clase del 1 er período, el $70,6 \%$ de las mujeres y el $81,9 \%$ de los hombres consumieron bebidas alcohólicas, mientras que para los estudiantes del $8^{\circ}$ período estas tasas respectivas fueron del $100 \%$ y del $80 \%$. Conclusión: La investigación reveló una alta prevalencia de consumo de alcohol, notablemente mayor para las mujeres a lo largo de la carrera médica.

Palabras clave: Consumo de alcohol, Estudiantes de medicina, AUDIT.

${ }^{1}$ Centro Universitário de Brasília (UniCEUB), Brasília - DF. *E-mail: rafaelaasmiranda@hotmail.com 


\section{INTRODUÇÃO}

O elevado consumo de bebidas alcoólicas é um problema mundialmente presente. A Organização Mundial de Saúde - OMS - define como consumo moderado de álcool a ingestão de uma dose/dia para as mulheres e duas doses/dia para os homens (WORLD HEALTH ORGANIZATION, 2000). Doses diárias acima destas taxas são prejudiciais, podendo representar algum risco para a saúde. Caso o consumo em um único evento seja elevado, é classificado como "o beber pesado episódico (BPE)", ou binge drink, que se caracteriza pelo consumo de mais de cinco doses para homens e quatro doses para mulheres em único episódio (LARANJEIRA R, et al., 2014). Alcoolismo é uma doença caracterizada pelo uso crônico e excessivo de álcool, que resulta em problemas psicológicos, interpessoais e médicos (KAPLAN HIMD e SADOCK BJ, 1993).

A entrada na universidade é considerada um período de mudanças, onde o estudante sofre alteração do seu estilo de vida, sendo a ingestão de álcool um hábito adquirido nesta fase da vida (ROCHA L, et al., 2011). O I Levantamento entre acadêmicos Nacional Sobre o Uso de Álcool, Tabaco e outras drogas entre Universitários, no qual colheu informações de 12.711 estudantes de 27 capitais brasileiras, revelou um consumo de álcool maior entre universitários do que a população geral, sendo que $22 \%$ dos entrevistados manifesta risco de dependência alcoólica e $36 \%$ apresenta um padrão binge drink. (ANDRADE A, DUARTE P e OLIVEIRA L, 2010)

Estudos demonstram que a maioria dos graduandos de medicina consome álcool (ABREU T, et al., 2018; PADUANI G, et al., 2008; PEDROSA A, et al., 2011). Fatores como a alta carga horária, grande quantidade de informações, cobrança dentro e fora do ambiente acadêmico, independência financeira tardia, privação do convívio familiar e do lazer propiciam o abuso alcoólico (ABREU T, et al., 2018; KENNETH H, et al., 2008). Além disso, o baixo custo, fácil acesso, campanhas publicitárias e elevada aceitação social são contribuintes (PEDROSA A, et al., 2011; MENDONÇA A, et al., 2018).

O elevado consumo etílico entres os estudantes de medicina é contraditório, uma vez que, na teoria, estes deveriam ser providos de conhecimento sobre os malefícios do álcool. Porém, estudos mostram que o ensino sobre este assunto, durante a formação acadêmica, é insuficiente, uma vez que a maioria das instituições não traz este tema na grade curricular (MESQUITA E, et al., 2008; LEMOS K, et al., 2007).

A alta ingesta de bebida alcoólica representa um fator de risco para comportamentos de risco à saúde, como beber e dirigir, riscos à atividade sexual, violência e suicídio (ANDRADE A, et al., 1997; FRANCA C e COLARES V, 2008; GALDURÓZ J, et al., 2005). Ademais, dentre os malefícios, tal hábito de vida pode gerar alterações nas relações com a família, colegas e professores, baixo rendimento escolar, agressões, crimes (STUEVE A e O'DONNELL LN, 2005). Tais adversidades afetam o desenvolvimento físico, emocional e social dos jovens, além da continuidade e qualidade de sua formação.

Assim, objetiva-se com este estudo estimar a prevalência do consumo de álcool entre estudantes de uma faculdade de Medicina no Distrito Federal.

\section{MÉTODOS}

Trata-se de um estudo transversal e descritivo, no qual foi realizada uma coleta de dados por meio da aplicação do questionário Alcohol Use Disorders Identification Test - AUDIT -, durante os meses de novembro e dezembro de 2018.

O AUDIT, já validado para a língua portuguesa, foi elaborado pela OMS, como ferramenta de rastreamento específico para verificar pessoas com uso abusivo do álcool (MÉNDEZ EB,1999). É um questionário constituído por dez questões. Três perguntas têm como objetivo avaliar a frequência e quantidade de consumo alcóolico, outras três exploram presença de sintomas de dependência e as demais questões averiguam as consequências do consumo de álcool que podem afetar a vida do entrevistado.

A depender da resposta do indivíduo, ele irá receber uma pontuação que pode variar de 0 a 40 pontos. A partir desta pontuação, então, pode-se classificar o entrevistado em "zonas de risco". Desta forma, pessoas que fazem uma pontuação de 0 a 7 se enquadram na zona I ou "padrão de beber de baixo risco". Pontuações de 8 a 15 classifica o indivíduo em zona II ou "padrão de médio risco". Aqueles com 16 a 19 pontos estão 
relacionados a zona III, onde há um uso nocivo. Por fim, pontuações de 20 a 40 refere à zona IV, demonstrando uma possível dependência ao uso de álcool. (MORETTI-PIRES RO e CORRADI-WEBSTER CM, 2011).

Para indicar o consumo de álcool, foi utilizado como método de definição a questão 1 do Audit: "com que frequência você consome bebidas alcoólicas", sendo qualquer resposta diferente de "nunca" considerado como possuir o hábito de beber. Enquanto o BPE foi estabelecido como qualquer resposta diferente de "nunca" para a questão 3 do AUDIT: "Qual a frequência com que você consome seis ou mais doses de bebida alcoólica em uma ocasião" (SILVEIRA C, et al., 2008).

O questionário foi aplicado para 109 estudantes, dentre os que estavam matriculados no $1^{\circ}$ ou $8^{\circ}$ semestre, no Centro Universitário de Brasília, por meio do Formulários Google, pelo link: https://goo.gl/forms/epSk9j2wqpeFdo193. Este link foi compartilhado aos alunos nas redes sociais, como Whatsapp e Instagram, por meio de grupos onde constam todos os alunos, e contato individual com alguns alunos. Além disso, foi feito uma divulgação do projeto e do link por meio de apresentação presencial dos pesquisadores na sala de aula do oitavo semestre. Garantiu-se a manutenção do anonimato e ressaltou-se que o preenchimento era voluntário. Os participantes assinaram o Termo de Consentimento Livre e Esclarecido antes da realização da coleta.

Para a análise de dados, foram utilizadas as ferramentas de planilhas do Aplicativo Microsoft Excel®. Em seguida, efetuou-se uma análise de tipo descritiva, com base nas frequências absoluta e relativa das variáveis investigadas. Alguns questionários não foram adequadamente preenchidos, pela ausência ou recusa do estudante. O material com as informações dos entrevistados ficará guardado sob a responsabilidade dos pesquisadores com a garantia de manutenção do sigilo e confidencialidade.

A pesquisa foi aprovada pelo Comitê de Ética em Pesquisa do Centro Universitário de Brasília - UNICEUB sob o Parecer $n^{\circ} 3.027 .980$.

\section{RESULTADOS}

Do total de 109 alunos que cursam o $1^{\circ}$ e $8^{\circ}$ semestres, $44(40,4 \%)$ responderam o questionário, sendo $28(63,3 \%)$ destes cursando o $1^{\circ}$ semestre e $16(36,3 \%)$ cursando o 8은 semestre.

Constatou-se que $36(81,9 \%)$ pessoas consumiam bebidas alcoólicas, sendo 21 (75\%) cursando o 1 o período e $15(93,8 \%)$ o 8 o período. Separando por sexo, $82,1 \%$ das mulheres e $81,3 \%$ dos homens bebem. Na turma do $1^{\circ}$ período, $70,6 \%$ das mulheres e $81,9 \%$ dos homens fazem consumo de bebidas alcoólicas, ao passo que para os alunos do $8^{\circ}$ período tais taxas foram de $100 \%$ para as mulheres e $80 \%$ para os homens.

Com relação à frequência de consumo de bebidas alcoólicas, $25 \%$ dos alunos do primeiro período marcaram que nunca bebem, enquanto $6,2 \%$ dos estudantes do oitavo marcaram esta opção. O que reflete o hábito de beber presente em $75 \%$ dos acadêmicos do $1^{\circ}$ período e $93,8 \%$ do $8^{\circ}$ período. De forma que $32,1 \%$ no 1 \% período diz beber uma vez por mês ou menos, enquanto a taxa do 8 o período é de $25 \%$. A opção de beber duas a quatro vezes por mês foi escolhida por $28,6 \%$ dos acadêmicos do $1^{\circ}$ semestre e $56,3 \%$ do $8^{\circ}$. Já $14,3 \%$ do $1^{\circ}$ e $12,5 \%$ do $8^{\circ}$ afirmam beber de duas a três vezes por semana. Em contrapartida, não houve resposta compatível com o beber quatro ou mais vezes por semana em ambas as turmas.

No que se refere a quantidade de bebidas contendo álcool consumida em um dia normal, os resultados da turma do 1ํ período foram: $46,4 \%$ dizem beber uma ou duas bebidas alcóolicas, 35,7\% bebem três ou quatro, $14,3 \%$ cinco ou seis e 3,6\% de sete a nove. Já na turma do 8 período, $37,5 \%$ afirma beber uma ou duas bebidas alcoólicas, $50 \%$ três ou quatro e $12,5 \%$ bebem cinco ou seis.

$50 \%$ dos alunos do $1^{\circ}$ semestre afirmam que nunca consumiram de seis bebidas ou mais em uma única ocasião, enquanto $32,1 \%$ relatam praticar tal ato menos de uma vez por mês e $17,9 \%$ praticam pelo menos uma vez por mês. Já os alunos do $8^{\circ}$ período, $37,5 \%$ diz nunca consumir de seis ou mais bebidas em uma única ocasião, 43,8\% consomem menos de uma vez por mês, $12,5 \%$ consomem pelo menos uma vez por mês e 6,2\% dizem consumir tal quantidade pelo menos uma vez por semana. Desse modo, podemos afirmar que $50 \%$ dos alunos do $1^{\circ}$ período bebem em binge drink, já entre os alunos do $8^{\circ}$ período o número sobe para $62,5 \%$ (Tabela 1). 
Tabela 1 - Presença de hábito de beber e BPE entre os estudantes entrevistados. Hábito de beber* $\mathrm{BPE}^{\star *}$

1ํ Período $\quad 75 \% \quad 50 \%$

8 Período $\quad 93,8 \% \quad 62,5 \%$

Legenda: * Aqueles que responderam qualquer resposta diferente de "nunca" para a questão 1 do AUDIT. ** Aqueles que responderam qualquer resposta diferente de "nunca" para a questão 3 do AUDIT.

Fonte: Miranda RAS, et al., 2020.

$96,4 \%$ dos alunos do $1^{\circ}$ período e $87,5 \%$ do $8^{\circ}$ período, afirmam que nunca se perceberem que não conseguiam parar de beber depois de começar nos últimos 12 meses. Entretanto, 3,6\% do 1 semestre e $12,5 \%$ do $8^{\circ}$ admitem ter tido essa percepção pelo menos uma vez por mês.

Considerando a situação de não conseguir cumprir as tarefas habitualmente exigidas por ter bebido, nos últimos 12 meses, 89,3\% dos alunos do 1ำ período afirmam que nunca ocorreu e 10,7\% diz que ocorreu menos de uma vez por mês. $93,8 \%$ da turma do $8^{\circ}$ semestre nunca passou por tal situação e em $6,2 \%$ da classe ocorreu menos de uma vez por mês.

Nos últimos 12 meses, 7,1\% dos alunos do 1ำ período precisou beber menos que uma vez ao mês pela manhã para se sentir bem ao longo do dia, após ter bebido no dia anterior. Na turma mais adiantada, ninguém passou por essa situação.

A maioria da turma do $1^{\circ}$ semestre $(64,3 \%)$ nunca sentiu culpa ou remorso depois de ter bebido neste último ano. 32,1\% tiveram estes sentimentos menos de uma vez por mês e 3,6\% sentiu pelo menos uma vez por semana. Já a turma do $8^{\circ}$ semestre, $81,3 \%$ nunca se sentiu culpado, enquanto $18,7 \%$ sentiu menos de uma vez ao mês.

$71,4 \%$ dos calouros nunca passaram pela situação de não se lembrar o que aconteceu devido a bebida no prazo de um ano. Contudo, $25 \%$ da turma passou por isso menos de uma vez por mês e $3,6 \%$ passou pelo menos uma vez por mês. Em $62,5 \%$ da turma do 8 을 semestre nunca ocorreu tal situação, em $25 \%$ ocorreu menos de uma vez por mês e em $12,5 \%$ aconteceu pelo menos uma vez por mês.

A maior parte da turma $(85,8 \%)$ matriculada no $1^{\circ}$ semestre nunca causou ferimentos ou prejuízos a si mesmo ou a outra pessoa após ter bebido. $7,1 \%$ já causou danos, porém não nos últimos 12 meses e os $7,1 \%$ restantes afirmam que este fato aconteceu no último ano. Com a turma do $8^{\circ}$ semestre, $6,3 \%$ dos estudantes ficou ferido ou feriu alguém, mas não nos 12 meses restantes. O restante da turma nega que tal fato tenha ocorrido.

$7,1 \%$ da turma do 1ำ período declarou que aconteceu, nos últimos 12 meses, de algum parente, amigo ou profissional de saúde manifestar preocupação pelo consumo de álcool ou sugerir que deixasse de beber. Com a turma do $8^{\circ}$ período, esta taxa é de $12,5 \%$.

De forma geral, a classificação de risco revelou que a maioria dos estudantes se enquadraram na Zona I, ou seja, consumo de baixo risco e a outra parte ficou na Zona II, sendo que não foram identificados alunos nas Zonas III e IV (Tabela 2).

Tabela 2 - Classificação dos estudantes dentro das Zonas de risco.

\begin{tabular}{cccc}
\hline & 10 Período & 8. Período & Geral \\
\hline Zona I & $85,7 \%$ & $87,5 \%$ & $86,4 \%$ \\
Zona II & $14,3 \%$ & $12,5 \%$ & $13,6 \%$ \\
Zona III & - & - & - \\
Zona IV & - & - & - \\
\hline
\end{tabular}

Fonte: Miranda RAS, et al., 2020. 


\section{DISCUSSÃO}

Com base neste estudo, constatou-se que cerca de $80 \%$ dos estudantes entrevistados do curso de medicina matriculados no $1^{\circ}$ e $8^{\circ}$ semestres consomem bebidas alcoólicas pelo menos uma vez por mês. Este dado é consonante com a maioria dos estudos já realizados em diferentes estados brasileiros, assim como pesquisas internacionais (ABREU T, et al., 2018; LEMOS K, et al., 2007; PINHEIRO M, et al., 2017; PADUANI G, et al., 2008; SHAKESHAFT A, et al., 1998; SINCLAIR J, et al., 2019; GARCÍA-CARRETERO M, et al., 2019; BOGOWICZ P, et al., 2018; MOUTINHO I, et al., 2019; OWENS B, 2018).

Em uma pesquisa realizada no Reino Unido cerca de 50\% dos estudantes de medicina do 30 ano da Universidade de Southampton apresentaram resultado positivo para o consumo de álcool de alto risco. Outro estudo da Espanha apontou que, apesar de majoritariamente apresentarem baixo risco para consumo de álcool, é comum a prática de binge drink, principalmente aos finais de semana (SINCLAIR J, et al., 2019; GARCÍA-CARRETERO M, et al., 2019).

O presente estudo expôs que apenas $13,6 \%$ dos entrevistados se enquadram na zona de risco II da classificação do AUDIT. Tal fato contrasta com um outro estudo realizado na Universidade do Reino Unido o qual traz dados sobre o consumo de álcool entre os estudantes de medicina e de direito. Ele demonstrou que mais da metade dos estudantes de medicina do primeiro $7(53,1 \%)$ e do segundo ano $(59,7 \%)$ tiveram uma pontuação do AUDIT maior ou igual a 8, que já representa um médio risco para uso abusivo do álcool (Zona II). Contudo, comparando as taxas, o consumo de álcool entre os estudantes de medicina ainda foi menor que o consumo entre os estudantes de direito (BOGOWICZ P, et al., 2018).

Já no Brasil, em uma universidade de Fortaleza, a pesquisa mostrou que $81,2 \%$ dos alunos de medicina fazem consumo atual de bebida alcoólica (CARNEIRO E, et al., 2012). Na UNESP, no ano de 1999, o uso de álcool variou de $80 \%$ a $92 \%$ na vida (KERR-CORREA F, et al., 1999). Em Salvador, encontraram $93 \%$ de uso na vida de bebidas alcoólicas, 87\% nos últimos 12 meses (LEMOS K, et al., 2007). Na universidade Federal de Uberlândia, observou-se que $66 \%$ dos entrevistados fazem uso de bebidas alcóolicas (PADUANI G, et al., 2008). $85 \%$ dos estudantes de todos os anos do curso de Medicina da UFMG fazem uso de álcool (PETROIANU A, et al., 2010).

Verificou-se que o consumo de álcool entre as mulheres $(82,1 \%)$ é ligeiramente maior que entre os homens $(81,3 \%)$, sendo esta diferença pouco significativa. Tal fato difere do exposto nos demais trabalhos realizados, onde existe uma predominância de consumo de álcool entre os homens (ABREU T, et al., 2018; GOMES L, et al., 2018; PADUANI G, et al., 2008; ROCHA L, et al., 2011; SILVEIRA C, et al., 2008).

Em um estudo realizado em Minas Gerais englobando duas faculdades de medicina, $76,6 \%$ dos estudantes fazem uso de bebidas alcoólicas, sendo o consumo maior entre homens, chegando a $83,7 \%$ (ABREU T, et al., 2018). Com relação a classificação de risco, baseado no questionário Audit, $73,3 \%$ dos estudantes foram classificados como "consumo de baixo risco", sendo o resultado deste presente trabalho semelhante, o qual a maioria dos estudantes também se enquadram na zona I de risco.

Dentre os possíveis fatores indutores ou protetores ao uso de álcool, Moutinho, em um estudo longitudinal, acompanhou 327 alunos durante 2 anos. Assim, observou-se que o uso anterior de substâncias está associado ao maior risco do alto consumo de álcool. Em contrapartida, a religião e a idade diminuem as chances de abuso de etílicos (MOUTINHO I, et al., 2019).

Embora o presente estudo tenha demonstrado poucos entrevistados que sofreram ou causaram danos pelo excesso de álcool, este tópico deve ser analisado com cautela, uma vez que refere a qualidade de vida de uma pessoa. Em uma publicação de Owens, o abuso de álcool foi fortemente associado a burnout, depressão, piores condições de vida e aumento de dívidas (OWENS B., 2018).

A relação entre o consumo do álcool e o suicídio foi relatado no estudo de Carrasco-Farfan (2019), Coentre, Faravelli e Figueira (2016) e Pinzón-Amado et al (2013), os quais detectaram uma forte associação de exposição e desfecho. Carrasco-Farfan expõe que isto decorre da elevada carga de estresse, dos maus tratos que os estudantes são constantemente expostos. Além disso, o abuso de álcool leva à diminuição da autoestima e à alguns transtornos mentais (ansiedade, depressão), aumentando, assim, o risco de suicídio (CARRASCO-FARFAN C, et al., 2019; COENTRE R, et al., 2016; PINZÓN-AMADO A, et al., 2013). 
Já o estudo de Ayala (2017) apontou que as consequências do uso de álcool e drogas entre os estudantes de medicina incluíram as altercações interpessoais, ideação suicida grave, déficits cognitivos, diminuição do desempenho acadêmico e condução de veículos sob influência de substâncias (AYALA E, et al., 2017).

Diversos estudos têm surgido em busca de minimizar o consumo de álcool entre os estudantes de medicina. No Reino Unido, um projeto liderado por St George's, da Universidade de Londres, possibilitou o desenvolvimento de diretrizes nacionais para a graduação médica, a respeito do uso indevido de substâncias (CARROLL J, et al., 2014).

Já em Israel, em busca de um programa de conscientização e aprendizado sobre o risco do uso indevido do álcool e a fim de diminuir a exposição aos risco os quais os estudantes são expostos; foi realizada uma intervenção com estudantes de medicina do quarto ano, os quais deveriam responder ao Questionário de Conhecimento dos Aspectos Psiquiátricos do Álcool (KPAAQ) previamente e após um ano de serem expostos a uma série de atividades lúdicas e educacionais que procurou promover o conhecimento sobre os Transtornos de Uso de Álcool. Observou-se que aqueles que participaram da intervenção obtiveram maiores pontuações que em relação aos estudantes que não passaram pela intervenção. Dessa forma, ficou evidente que a atividade foi efetiva de modo que o conhecimento foi consolidado após um ano (JAWOROWSKI, S., 2018).

Diante dos resultados do presente estudo, assim como os demonstrados na literatura, constata-se que a ingestão de bebidas alcoólicas entre os estudantes de medicina atinge altas taxas, exigindo uma maior cautela das universidades e autoridades, por meio do incentivo de campanhas de conscientização dos malefícios do uso excessivo de bebidas alcoólicas, criação de programas estudantis que eduquem e estimule um consumo responsável de bebidas alcoólicas.

\section{CONSIDERAÇÕES FINAIS}

Esta pesquisa avaliou a prevalência do consumo de álcool entre estudantes de uma faculdade particular de Medicina no Distrito Federal. Observou-se que $81,9 \%$ dos entrevistados fazem consumo de bebidas alcoólicas. Desta população, foi constatado uma maior frequência entre as mulheres. Observou-se também que o consumo de bebidas alcoólicas em estudantes de medicina tende a aumentar com o avançar do curso. O padrão em binge drink esteve presente na maior parte dos nossos entrevistados. Contudo, a maioria dos entrevistados apresentou-se na zona de risco I (padrão de baixo risco) da classificação do AUDIT. Além disso, os estudantes que participaram dos estudos, em sua generalidade, negaram que já tenham sofrido algum dano ou malefício decorrente da ingestão de álcool.

\section{AGRADECIMENTOS}

Agradecemos primeiramente a Deus, pois nada seríamos sem a ajuda Dele. Agradecemos ao Doutor e Orientador Antônio Garcia pelo suporte, correções e incentivo. Agradecemos também aos colegas de Universidade que aceitaram participar da pesquisa. Por fim, gostaríamos de agradecer aos familiares e amigos por sempre acreditarem em nós.

\section{REFERÊNCIAS}

1. ABREU T, et al. O consumo de bebida alcoólica e o binge drink entre os graduandos de Medicina de uma Universidade de Minas Gerais. Jornal brasileiro de psiquiatria, 2018. 67 (2): 87-93.

2. ANDRADE A, et al. Fatores de risco associados ao uso de álcool e drogas na vida, entre estudantes de medicina do Estado de São Paulo. Rev ABP-APAL, 1997. 19:117-26.

3. ANDRADE A, DUARTE P, OLIVEIRA L. I Levantamento nacional sobre o uso de álcool, tabaco e outras drogas entre universitários das 27 capitais brasileiras. Brasília, 2010.

4. AYALA E, et al. Prevalence, perceptions, and consequences of substance use in medical students. Med. Educ. Online, $2017 ; 22(1): 1392824$.

5. BOGOWICZ P, et al. Alcohol and other substance use among medical and law students at a UK university: a crosssectional questionnaire survey. Postgraduate Medical Journal. 2018; 94:131-136.

6. CARNEIRO E, et al. Fatores associados a beber pesado episódico entre estudantes de medicina. Rev. bras. educ. med., 2012; 36 (4): 524-530. 
7. CARRASCO-FARFAN C, et al. Alcohol consumption and suicide risk in medical internship: A Peruvian multicentric study. Drug and Alcohol Review, 2019.

8. CARROLL J, et al. Substance misuse teaching in undergraduate medical education. Bmc Medical Education, $2014 ; 14$ (1): 14-34.

9. COENTRE R, et al. Assessment of depression and suicidal behaviour among medical students in Portugal. Int. J. Med. Educ., 2016; 7:354-63.

10. FRANCA C, COLARES V. Comparative study of health behavior among college students at the start and end of their courses. Rev Saúde Pública, 2008. 42:1-7.

11. GALDURÓZ J, et al. Uso de drogas psicotrópicas no Brasil: pesquisa domiciliar envolvendo as 107 maiores cidades do país. Rev Latinoam Enferm, 2005; 13:888-95.

12. GARCÍA-CARRETERO M, et al. Patrones de consumo de alcohol en estudiantes universitarios de Ciencias de la Salud. Enfermería Clínica, 2019; 29 (5): 291-296.

13. GOMES L, et al. Consumo de álcool entre estudantes de medicina do Sul Fluminense - RJ. Rev Med (São Paulo), 2018; 97(3):260-6.

14. JAWOROWSKI, S. Preliminary results of a controlled educational intervention on alcohol related harm among medical students with a 12-month follow-up. Isr. J. Psychiatry, Jerusalem, 2018; 55 (2).

15. KAPLAN HIMD, SADOCK BJ. Compêndio de Psiquiatria, Ciências comportamentais, Psiquiatria Clínica. Porto Alegre: Artes médicas; 1993; 943p.

16. KENNETH H, et al. Social Context of Drinking and Alcohol Problems Among College Students. Am J Health Behav, 2008. 32:420-30.

17. KERR-CORREA F, et al. Uso de álcool e drogas por estudantes de medicina da Unesp. Rev. Bras. Psiquiatr, 1999; $21(2): 95-100$.

18. LARANJEIRA R, et al. II Levantamento Nacional de Álcool e Drogas (Lenad), São Paulo. Instituto Nacional de Ciência e Tecnologia para Políticas Públicas de Álcool e Outras Drogas (Inpad), Unifesp; 2014.

19. LEMOS K, et al. Uso de substâncias psicoativas entre estudantes de Medicina de Salvador (BA). Rev Psiquiatr Clín, 2007. 34(3):118-24.

20. MÉNDEZ EB. Uma Versão Brasileira do AUDIT (Alcohol Use Disorders Identification Test). Dissertação (Mestrado em Epidemiologia) - Faculdade de Medicina, Universidade Federal de Pelotas, Rio Grande do Sul, 1999; 86p.

21. MENDONÇA A, et al. Fatores Associados ao Consumo Alcoólico de Risco entre Universitários da Área da Saúde. Revista Brasileira de Educação Médica, 2018. 42 (1): 205-213.

22. MESQUITA E, et al. Avaliação das atitudes dos estudantes de medicina frente ao abuso de drogas por colegas do meio acadêmico. Rev Psiquiatr Clín, 2008. 35(1):8-12.

23. MORETTI-PIRES RO, CORRADI-WEBSTER CM. Adaptação e validação do Alcohol Use Disorder Identification Test (AUDIT) para população ribeirinha do interior da Amazônia, Brasil. Cad. Saúde Pública, 2011; 27 (3): $497-509$.

24. MOUTINHO I, et al. Prevalence, Incidence, and Factors Associated With Substance Use Among Medical Students: A 2-Year Longitudinal Study. J Addict Med. 2019; 13(4):295-299.

25. OWENS B. Growing concern over medical students' excessive drinking. CMAJ, 2018; 190(40): E1215.

26. PADUANI G, et al. Consumo de álcool e fumo entre os estudantes da Faculdade de Medicina da Universidade Federal de Uberlândia. Rev. bras. educ. med., 200832 (1): 66-74.

27. PEDROSA A, et al. Consumo de álcool entre estudantes universitários. Cad. Saúde Pública, 2011. 27(8):1611-1621.

28. PETROIANU A, et al. Prevalência do consumo de álcool, tabaco e entorpecentes por estudantes de medicina da Universidade Federal de Minas Gerais. Rev Assoc Med Bras. 2010; 56(5):568-571.

29. PINHEIRO M, et al. Prevalência e Fatores Associados ao Consumo de Álcool e Tabaco entre Estudantes de Medicina no Nordeste do Brasil. Rev. Bras. de Educação Médica, 2017; 41 (2): 231-250.

30. PINZÓN-AMADO A, et al. Suicide ideation among medical students: prevalence and associated factors. Ver. Colomb. Psiquiatr., 2013; 42:47-55.

31. ROCHA L, et al. Consumo de álcool entre estudantes de faculdades de Medicina de Minas Gerais, Brasil. Rev. bras. educ. med., 2011; 35 (3): 369-375.

32. SHAKESHAFT A, et al. Comparison of three methods to assess binge consumption: one week retrospective drinking diary, audit, and quantity/frequency. Substance Abuse,1998; 19 (4): 191-203.

33. SILVEIRA C, et al. Epidemiologia do beber pesado e beber pesado episódico no Brasil: uma revisão sistemática da literatura. Rev. psiquiatr. clín., 2008; 35 (1): 31-38.

34. SINCLAIR J, et al. Impact of Personal Alcohol Consumption on Aspects of Medical Student Alcohol-Related Competencies. Alcohol And Alcoholism, 2019; 54 (3): 325-330.

35. STUEVE A, O'DONNELL LN. Early alcohol initiation and subsequent sexual and alcohol risk behaviours among urban youths. Am J Public Health, 2005. 95(4):887-93.

36. WORLD HEALTH ORGANIZATION. International guide for monitoring alcohol consumption and related harm. Geneva: WHO, 2000. 